\title{
Inhalt von Band 217
}

\section{Heit 1/2}

\author{
Ausgegeben im Allgust 1961
}

N. I. Kobosezo, W. P. Lebedew und A.N. Malzew, Die Bestimmung der Zusammensetzung, Zahl und absoluten Wirksamkeit katalytisch aktiver Zentren. Mit $16 \mathrm{Ab}$. bildungen und 8 Tabellen. (Eingegangen am 11. 5. 1960) .............

E. Lange. Allgemeine Grundbegriffe der elektrochemischen Kinetik. Mit 2 Abbildungen.

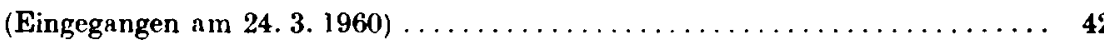

K. Altenburg, Schallgeschwindigkeit und Molekülstruktur. IV. Schallgeschwindigkeit und molekulare Daten. Mit 3 Abbildungen und 1 Tabelle. (Eingegangen am 31. 5. 1960) .........................................

R. Havemann. W. Haberditzl und P. Grzegorzewski. Ober den Diamagnetismus des Porphyrinsystems. Mit 2 Abbildungen und 10 Tabellen (Eingegangen am

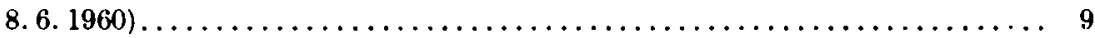

E. A. Niekisch, Ein Beitrag zur Frage der Störterme und ihrer Natur in Cadmiumsulfid. Mit 9 Abbildungen und 3 Tabellen. (Eingegangen am 19. 11. 1960)

\section{Kurze Mitteilung}

$J$. Richter und $W$. Lorenz, Vergleichende radiochemische und elektrochemische Studie zur Ionenadsorption an Platin

\section{Buchbesprechumien}

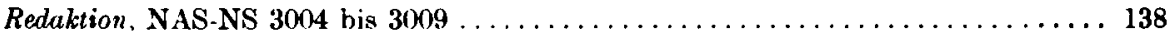

Redaktion, Angular Scenttering functions for Spherical Particles $\ldots \ldots \ldots \ldots \ldots 138$ 


\section{Heft 3/4}

Ausgegehen im September 1961

E. Herczynaka, Deposition of ${ }^{a 5} \mathrm{Zn}$ and ${ }^{32} p$-Phosphate on Various Metallic and NonMetallic Surfaces. With 22 figures. (Received 13. 6. 1960) . . . . . . . . . . . 139

J. Auth. Untersuchungen über den Photo-Magneto-Elektrischen Effekt und die Eigenschaften der Iöcher in CdS. Mit 12 Abbildungen und 4 Tabellen. (Eingegangen

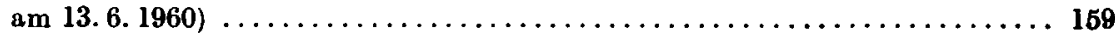

J. A uth, Eine Spekulation über die Rolle ,heißer“ Elektronen beim , anomalen“ PEMEffekt. (Eingegangen am 15. 6. 1960) $\ldots \ldots \ldots \ldots \ldots \ldots \ldots \ldots \ldots \ldots \ldots \ldots 188$

H. Schiller, Die Wechselwirkung der Redox-Systeme im Rinderhämoglobin. Mit $1 \mathrm{Ab}$ bildung und 2 Tabellen. (Eingegangen am 24. 6. 1960) .............. 194

D.K. Majumdar, Quenching of Fluorescence of Dyes by Neutral Salts. With 1 table. (Received 12.6. 1960) .............................. 200

H. Sadek. Density Variation During Copper Deposition. With 5 figures and 3 tables. (Received 6. 7. 1960) ................................ 207

E. Leibnitz und C.Schuhler. Berechnung von Destillationskolonnen bei Vielstoffgemischen, Teil I. Mit 3 Tabellen. (Eingegangen am 26. 7. 1960) . . . . . . 217

F. Gutmann, On the Temperature Dependence of the Permittivity of Dielectrics. (Received 19. 7. 1960) ................................ 230

H. Schuberth. Vorausberechnung von Dampf-Flüssigkeits-Phasengleichgewichten heteroazeotroper binärer Systeme mit relativ geringer gegenseitiger Löslichkeit und Prüfung derselben an experimentellen Daten. I. Mit 2 Abbildungen und 1 Tabelle. (Eingegangen am 21. 7. 1960) $\ldots \ldots \ldots \ldots \ldots \ldots \ldots \ldots \ldots \ldots$

K. Palm und H. Dunken, Dipolmomentbestimmungen an assoziierten o-Halogenbenzoesäuren und Benzoesäure in Benzol. Mit 3 Abbildungen und 1 Tabellé. (Eingegangen am 20. 9. 1960)

C. Schuhler, Berechnung von Destillationskolonnen bei Vielstoffgemischen, Teil 2. Mit I Abbildung. (Eingegangen am 10. 10. 1960) .................. 263

\section{Buchbesprechungen}

U. Drehmann, Travaux Pratiques de Physique Nucléaire et de Radiochimie, v.M. Duquesne, R. Grégoire und M. Lefort. ...................... 271

O. Hauser, Graphite and its Crystal Compounds, v. A. R. Ubbelohde und F. A. Lowis 271 


\section{Heft $5 / 6$}

fusgesuben im Oktoher 190 il

II. Hrapia und E. Leibuitz, Zur Trennstufenzahl von Chromatographiesïulen. Mit 9 Abbildungen und 5 Tabellen. (Eingegangen am 30. Juli 1960) ..........

V. G.Voicu. Beiträge zum elektrochemischen Studium des Thalliums in hoch verdünnten Lösungen. Mit 2 Abbildungen und 3 Tabellen. (Eingegangen am

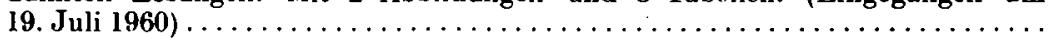

A. Terenin and I. Akimov, On the Mechanism of the Optical Sensitization of Semiconductors by Organic Dyes. With 2 figures and 2 tables. (Received 8. August 1960) 307

$R$. Jaeckel und W. Peperle, U'ber die Abhängigkeit des Verdampfungskoeffizienten vom Partialdruck über der abdampfenden Kristallflāche. Mit 16 Abbildungen und 8 Tabellen. (Eingegangen am 10. August 1960) ................ 32

G. Vojta. Zur statistischen Thermodynamik von idealen Gasen mit isotopensubstituierten Molekülen. Mit 6 Tabellen. (Eingegangen am 22. August 1960) . . . 337

K. K. Rohatgi. The Fluorescence Life-times of Fluorescein Anion. With 4 tables. (Received 26. July 1960) ................................ 353

B. Lengyel und Z. Boksay, Úber die elektrische Leitfähigkeit des Glases. IV. (Gläser mit zwei verschiedenen zweiwertigen Kationen. Mit 7 Abbildungen und 2 Tabellen. (Eingegangen am 19. Juli 1960) ........................ 357

R. Landsberg, V. Stockmann und W. Geißler, Zum polarographischen Verhalten von Zinkatlösungen. Mit 3 Abbildungen und 2 Tabellen. (Eingegangen am

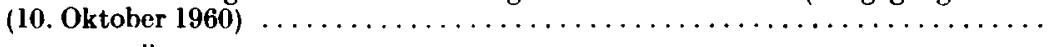

H. IIartmann, Uber die Adsorptionsmischkristalle zwischen organischen Farbstoffen und Bleiacetat-Trihydrat. Mit. 8 Abbildungen und 4 Tabellen. (Eingegangen am

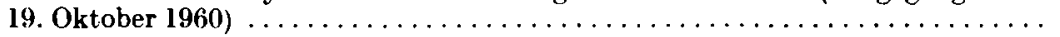

V. Armeanu und C. Luca, Die Wirkung der Substituenten im Ammoniakmolekül auf die Beständigkeit der mit den entsprechenden Aminen gebildeten komplexen Silberionen. II. Die Beständigkeit der mit einigen aliphatischen Aminen und Alkylanilinen gebildeten kompiexen Silberionen. Mit 14 Tabellen. (Eingegangen

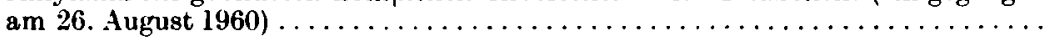

(1. Ohlmann und E. Leibnitz, Studium der Kinetik und des Mechanismus der Oxydation von Benzinkohlenwasserstoffen in der Gasphase. I. Mitteilung: Kurze Literaturübersicht ïber den 'Tjeftemperaturmechanismus der Oxydation von höheren Kohlenwasserstoffen und über die Tieftemperaturoxydation von

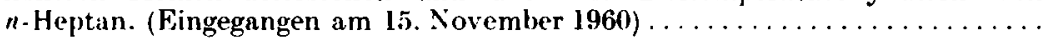

\section{Kurze Mitteilung}

Z. Fyulai. Kurze Bemerkungen zu den Kristallwachstumsformen. Mit 3 Abbildungen. (Eingegangen am 14. Februar 1961 ) ...................... 42

\section{Buchbesprechungen}

W. Eschke. Biophysical Science - A Study Program, v. J. L. Oncley............ 432

W. Brauer. Introduction to Statistical Thermodynamics, v. Terell L. Hill . . . . . 432

$K$. Schwabe, Kurzes Lehrbuch der physikalischen Chemie, v. H. Ulich . . . . . . 433

$K$. Schwabe, Electrochimie Théorique, v. F. Darmois und (. Darmois .......... 433

B. Eckstein, Struktur und physikalische Eigenschaften der Moleküle. v. M. W. Wolken-

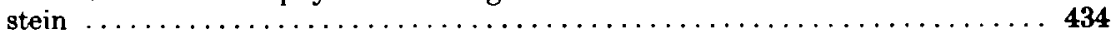

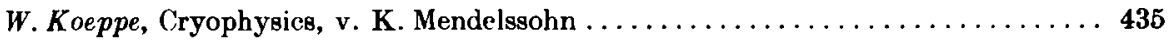

K. Th. Wilke, Frozen Free Radicals. v. G. J. Minkoff................ 436

R. v. Kujawa, Röntgenographische ('hemie, v. E. Brandenberger und W. Epprecht 437

H. G. Thom, Structure and Propertie's of Thin Films. v. C. A. Neugebauer, J. B. New-

kirk und D. A. Vermilyea ......................... 437 